\title{
FEN FERTILIZATION WITH POTASH IN THE LIGHT OF TESTS AT LETEENSUO EXPERIMENTAL STATION
}

\author{
YRJÖ PESSI \\ Society of Peat Cultivation, Experimental Station Leteensuo
}

Received July 28, 1960

Numerous experiments intended to clarify the fertilizing questions of peat land have been established at the Experimental Station of Leteensuo and carried on through fairly long periods without interruption. Short-time reports of these experiments have been given in several connections. The results obtained over longer periods have recently been analysed with reference to the Sphagnum bog fertilizing tests, which have thrown light on the effects of nitrogen and phosphorus as well as potassium fertilization $(5,6)$. Moreover, TAKALA $(6)$ has reported on the experiments arranged with a view to clarifying the effects of phosphate fertilization. In the following, the significance of potassium fertilization on the fen area of the Experimental Station of Leteensuo will be described on the basis of the results derived from three field tests. The persons supervising the experiments in different years have been mentioned in a previous publication $(4$, p. 2$)$. With reference to shorter periods, these tests have been analysed by MaLm (3) and HiRvensalo (2). The author has previously examined the results of one of these tests over a longer period (5).

\section{Test ground}

The peat of the areas is forest sedge peat and their bog type prior to clearing was that of a wooded swamp with herbs and grasses. The depth of the peat layer is more than $2 \mathrm{~m}$. The test areas are drained by open ditches dividing the land into strips $20 \mathrm{~m}$ in width.

The quality of the peat is illustrated by the chemical analyses made from the plot adjacent to the site of claying test No. 1 ( 7 , p. 32 ; 8$)$. The chemical composition of the clay and sand used as soil improving agents and the results of the soil analysis can also be found in the said publication by VESIKIVI $(7$, p. 34). 
Test No. 1. - The test area was cleared for cultivation in 1904. In 1905 clay was added as soil improving agent in a quantity of $400 \mathrm{~m}^{3}$ per hectare. The test was started in 1905 . The treatments were: $0, \mathrm{P}\left(40 \mathrm{~kg} \mathrm{P}_{2} \mathrm{O}_{5}\right.$ per hectare $), \mathrm{K}(100 \mathrm{~kg}$ $\mathrm{K}_{2} \mathrm{O}$ per hectare), $\mathrm{PK}$, and $\mathrm{PK}$ plus liming. The location of the test plots can be seen from Fig. 1. Liming was done as follows: in 1905, 2 tons slaked lime per hectare; in 1923, 1927 and 1933, 1 ton per hectare each. Fertilization was carried out according to plan during the period of 1905-1918. In the years 19191922 all test plots were given phosphate and potassium fertilizer. From 1923 onwards, fertilization again proceeded according to the original test plan, but the fertilizer quantities given to root crops were doubled.

Test No. 2. - The test area is located adjacent to that of

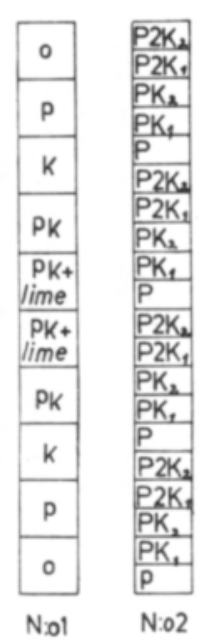

Fig. 1. The position of plots in the tests No. 1 and 2 . Test No. 1. No clay was added. The test was started in 1933, but its test plan was altered in 1937. Initially, it consisted of the following treatments: $\mathrm{P}\left(\mathrm{P}=40 \mathrm{~kg} \mathrm{P}_{2} \mathrm{O}_{5}\right.$ per hectare), $\mathrm{PK}_{1}\left(\mathrm{~K}_{1}=\right.$ $60 \mathrm{~kg} \mathrm{~K} \mathrm{~K}_{40}$ per hectare), $\mathrm{PK}_{2}\left(\mathrm{~K}_{2}=60 \mathrm{~kg} \mathrm{~K} \mathrm{~K}_{20}\right.$ per hectare), $\mathrm{P} 2 \mathrm{~K}_{1}$, and $\mathrm{P} 2 \mathrm{~K}_{2}$. In 1937, $\mathrm{K}_{40}$ was replaced with the equivalent quantity of potassium sulphate and $\mathrm{K}_{20}$ with the equivalent quantity of $\mathrm{K}_{40}$. The location of the test plots can be seen from Fig. 1.

Test No. 3. - Cultivation of the test area was commenced in 1921. One half of the test strip was clayed (adding $200 \mathrm{~m}^{3}$ clay per hectare) in 1923 and in 1941. The potassium fertilizer quantities applied at first were: $0,20,40,60$, and $80 \mathrm{~kg} \mathrm{~K}_{2} \mathrm{O}$ per hectare; in 1930 they were replaced by the following series: $0,30,60$, 90 , and $120 \mathrm{~kg}$ per hectare (see 5, p. 4). The notations employed in this paper with respect to the treatments in this test refer to the latter series. The entire area has been fertilized annually with phosphate fertilizer equivalent to about $300 \mathrm{~kg}$ superphosphate per hectare.

\section{Crop yield results}

The weather conditions during the test years have been described in a previous publication (4).

The annual crop yields in tests No. 1 and 2 can be seen from Tables I and II in the Appendix. The corresponding figures relating to test No. 3 have been published before (5).

Meadows. - The average annual hay crops obtained in the tests during the test period are shown in Tables $1-3$ and in Fig. 2. Table 1 reveals that potassium fertilization has increased the hay crop yield, this increase being significant at a level of $95 \%$. However, the increase in crop yield obtained in the case of the PK treatment shows that potassium fertilization in combination with phosphorus 
Table 1. Test No. 1. Average annual hay crop yields (during 19 years), kg per hectare.

Treatment

$\begin{array}{cc}\text { O } & 2010 \\ \text { P } & 4840 \\ \text { K } & 2770 \\ \text { PK } & 6510 \\ \text { PK }+ \text { lime } & 6640\end{array}$

Increase in yield

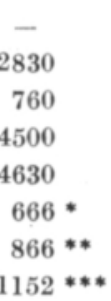

had a remarkable compound effect; the crop yield increases produced by $\mathrm{P}$ and $\mathrm{K}$ individually add up to $3590 \mathrm{~kg}$ per hectare, whereas their use in combination increased the crop yield by $4500 \mathrm{~kg}$ per hectare.

The test plan of test No. 2 did not include a plot without treatment. The fertilizing effect of potassium alone is therefore unknown. However, Table 2 reveals that the use of potassium in addition to phosphorus has a remarkable effect. Potas-

Table 2. Test. No. 2. Average annual hay crop yields (during 8 years), kg per hectare.

\begin{tabular}{ccc}
\hline Treatment & Crop yield & Increase in yield \\
\hline $\mathrm{P}$ & 3900 & - \\
$\mathrm{PKsO}_{4}$ & 6100 & 2200 \\
$\mathrm{PK}_{48}$ & 6390 & 2490 \\
$\mathrm{P}_{4} \mathrm{KsO}_{4}$ & 6540 & 2640 \\
$\mathrm{P}_{42}$ & 6850 & 2950 \\
& & $566 *$ \\
& & $762 * *$ \\
& & $1010 * * *$
\end{tabular}

sium sulphate was somewhat less efficient than potassium salt, but there are no statistically significant differences between the effects of the different fertilizer types. Doubled potassium quantity, on the other hand, increased the crop yield with reference to that obtained with the single quantities in a degree approaching statistical significance.

No plot without treatment was included in test No. 3 either. It can be seen from the results shown in Table 3 and Fig. 2 that potassium fertilization increased the hay crops considerably from those obtained in the $\mathrm{P}$ treatment. On the unclayed test strip, the increase was higher than on the clayed strip in the instance of the first four potassium fertilizer quantities in the series, but the highest potassium fertilizer addition produced about equal crop yield increases on both strips. This serves to show that clay has a considerable potassium fertilizing effect (cf. 1) but also other effects which cannot be reproduced with potassium fertilization. 
Table 3. Test. No. 3. Average annual hay crop yields (during 23 years), kg per hectare.

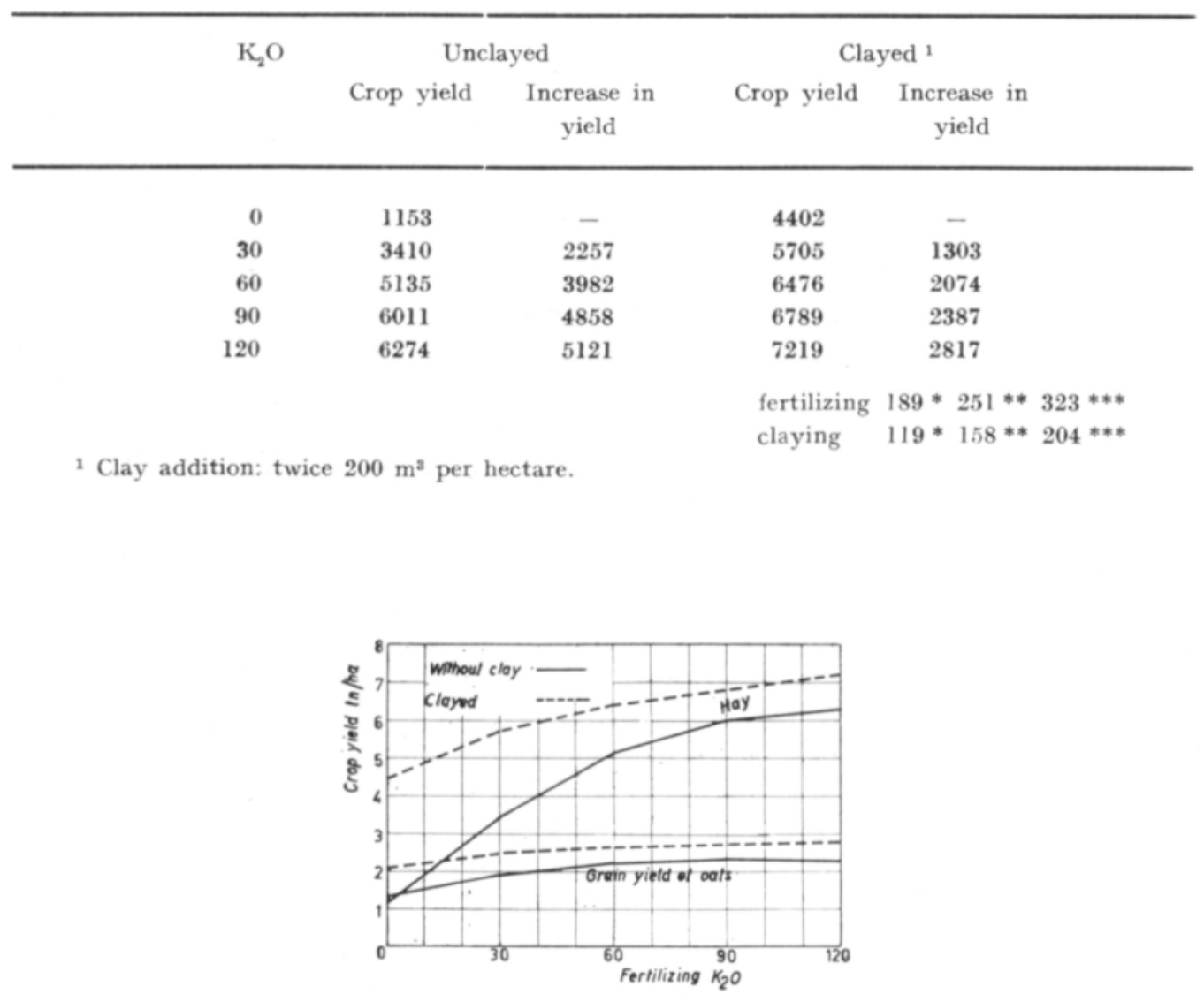

Fig. 2. Average annual hay crop yields and grain yield of oats in the test No. 3 .

Cereals. - In likeness with the hay crop yields, the cereal crop yields have been presented in Tables 4-6 and in Fig. 2. It can be seen that potassium fertilization has resulted in an increased grain yield of oats in tests No. 1 and 3, while in test No. 2 potassium fertilization tended to cause a slight decrease in the crop yield, although there are no statistically significant differences. Nor can any effect of potassium fertilization on the grain yield of spring wheat be seen in the same test. On the other hand, the straw yield was increased also in this test. Possibly this is due to inundations, which occurred repeatedly in the spring several years running and which may have transported such quantities of mud to the test area that adequate potassium fertilizer action has been produced.

Root crops. - Root crops were cultivated during several years on the test area of test No. 1 only. The results reveal that potassium alone does not produce much of an increase in the crop yield. Potassium in addition to phosphorus has a remarkable effect. 
Table 4. Test No. 1. Average annual grain and straw crop yields of oats (12 years) and barley ( 7 years), kg per hectare.

\begin{tabular}{|c|c|c|c|}
\hline Treatment & Grain yield & Incr. in grain yield & Straw yield \\
\hline \multicolumn{4}{|c|}{ Oats } \\
\hline $\mathrm{O}$ & 1230 & - & 2180 \\
\hline $\mathrm{P}$ & 2630 & 1400 & 4300 \\
\hline K & 1490 & 260 & 2510 \\
\hline PK & 3000 & 1770 & 5330 \\
\hline $\mathrm{PK}+$ lime & 2860 & 1630 & $5050^{\circ}$ \\
\hline \multicolumn{4}{|c|}{$285^{*}$} \\
\hline \multicolumn{4}{|c|}{$381 *$} \\
\hline \multicolumn{4}{|c|}{$501 * * *$} \\
\hline & & ley & \\
\hline $\mathrm{O}$ & 560 & - & 2130 \\
\hline $\mathrm{P}$ & 1320 & 760 & 3900 \\
\hline K & 820 & 260 & 2700 \\
\hline PK & 1740 & 1180 & 4810 \\
\hline PK + lime & 1940 & 1380 & 5010 \\
\hline \multicolumn{4}{|c|}{$506 *$} \\
\hline \multicolumn{4}{|c|}{$685 * *$} \\
\hline \multicolumn{4}{|c|}{$918 * * *$} \\
\hline
\end{tabular}

Table 5. Test No. 2. Average annual grain and straw crop yields of oats (5 years) and spring wheat (2 years), kg per hectare.

Treatment Grain yield Incr. in grain yield Straw yield

Oats

$\begin{array}{lrrr}\mathrm{P} & 2680 & - & 4310 \\ \mathrm{PKso}_{4} & 2530 & -150 & 4950 \\ \mathrm{PK}_{48} & 2670 & -10 & 5260 \\ \mathrm{P}_{4} \mathrm{Kso}_{4} & 2600 & -80 & 4950 \\ \mathrm{P}_{4} & 2590 & -90 & 5090 \\ & & & \\ & & \text { Spring wheat } & 4380 \\ \mathrm{P} & 1960 & - & 4700 \\ \mathrm{PKso}_{4} & 1960 & 120 & 4820 \\ \mathrm{PK}_{48} & 2080 & -90 & 4940 \\ \mathrm{P}_{4} K_{40} & 1870 & 80 & 4800 \\ \mathrm{P}_{4} & 2040 & & \end{array}$

\section{Conclusions}

On the fen area of the Experimental Station of Leteensuo, with a peat layer consisting of forest sedge peat, potassium fertilization produced a considerable increase of the hay crop yield on the clayed as well as the unclayed bog, when phosphate fertilization had been added. However, clay displayed a considerable 
Table 6. Test No. 3. Average annual grain and straw yields of oats (during 7 years), kg per hectare.

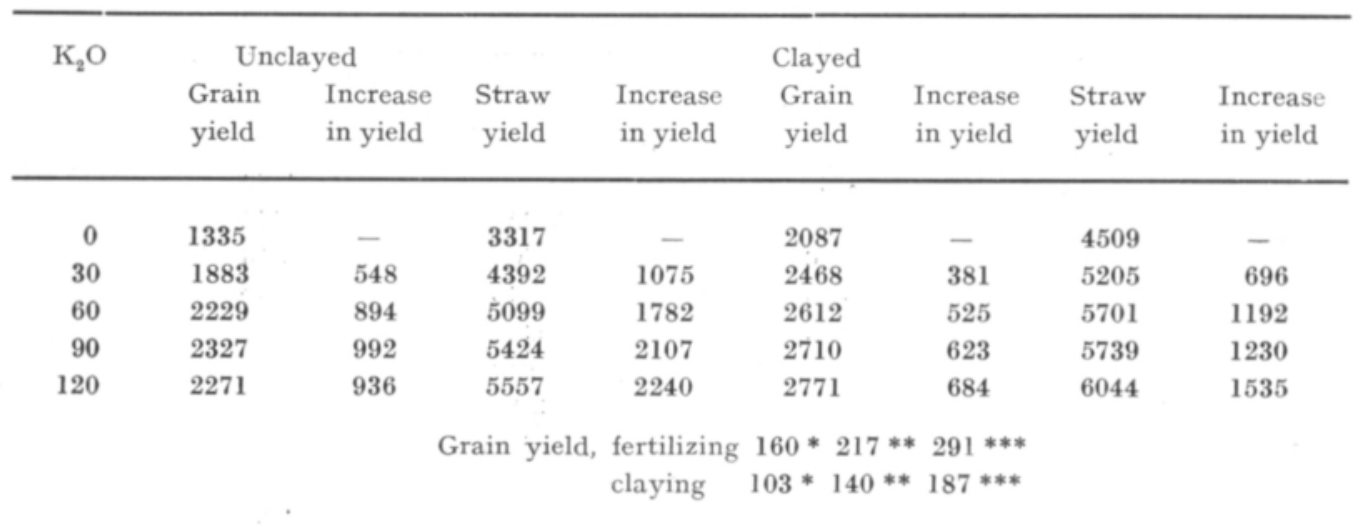

Table 7. Test No. 1. Average annual root and top yields of swede (during 4 years), kg per hectare.

\begin{tabular}{cccc}
\hline Treatment & Root yield & Incr. in root yield & Top yield \\
\hline & & & 2530 \\
P & 5230 & 16310 & 10560 \\
K & 21540 & 1070 & 2650 \\
PK & 29850 & 24620 & 11840 \\
PK + lime & 32480 & 27250 & 13190
\end{tabular}

potassium fertilizing effect and also other effects, which probably cannot be reproduced by fertilization.

The effect of potassium fertilization on the summer cereal crop yield was different in different tests. In two of them, potassium fertilization increased the grain yield but not in the third. The results obtained in this latter instance are thought to have been influenced by the addition of mud through the action of floods.

Potassium fertilization had remarkably increased the root crop yields.

When phosphate fertilization had not been added, the effect of potassium fertilization was small asa rule.

There was no marked difference between the effects of potassium salt and potassium sulphate, although it should be noted that the tests did not contain any treatments with potatoes as the experimental crops, which might show up this difference most clearly. 
Appendix I. Annual crop yields in test No. 1, kg per hectare $\times 10^{-1}$.

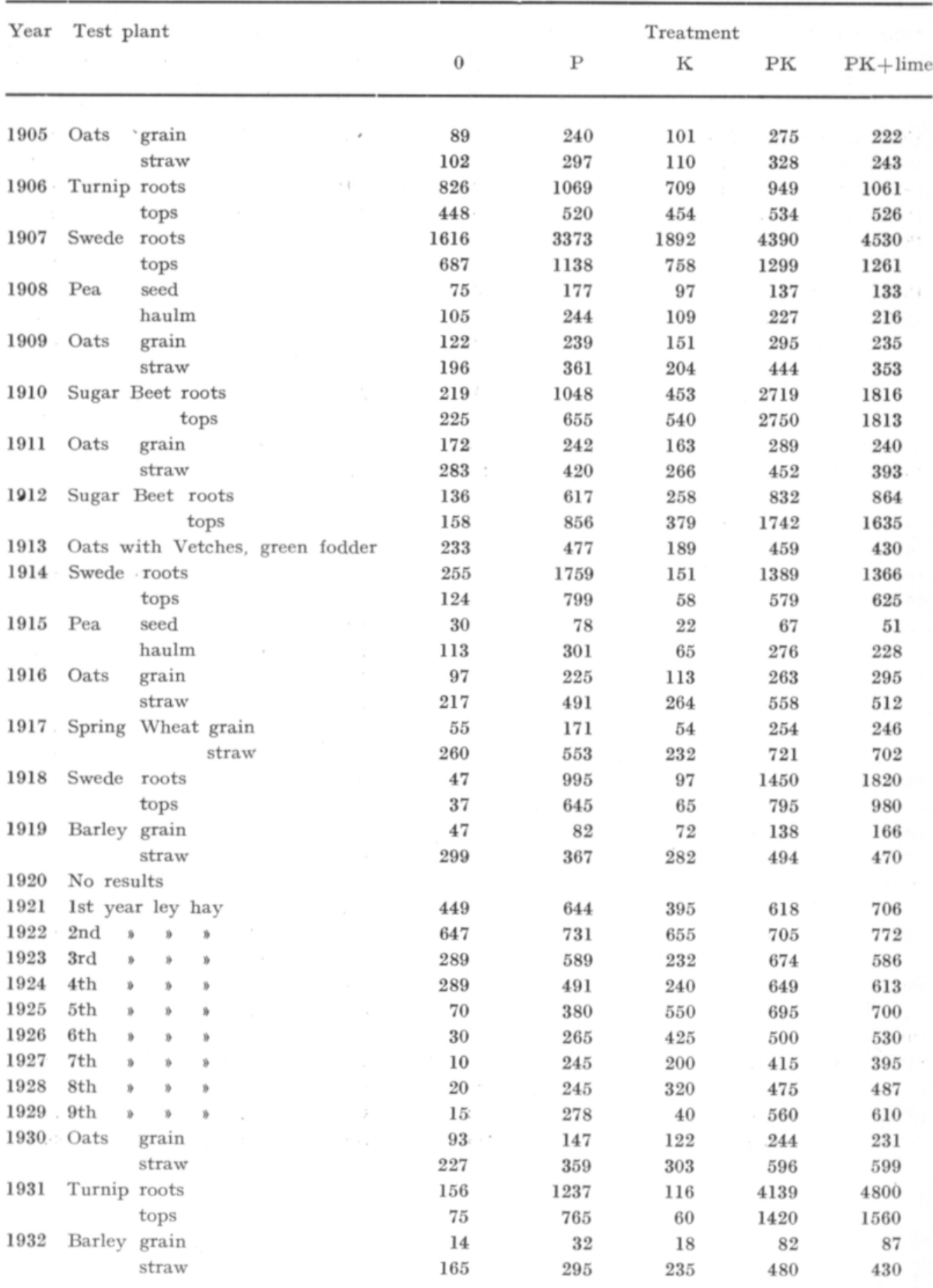


Year Tes plant

\section{Treatment}

$\begin{array}{lllll}0 & \text { P } & \text { K } & \text { PK } & \text { PK+lime }\end{array}$

\begin{tabular}{|c|c|c|c|c|c|c|c|c|}
\hline 1933 & lst yea & ar ley $h$ & hay & 155 & 420 & 255 & 780 & 745 \\
\hline 1934 & 2nd & , & . & 90 & 453 & 108 & 735 & 720 \\
\hline 1935 & 3rd , & , & , & 91 & 372 & 119 & 720 & 619 \\
\hline 1936 & 4th & , & $\cdot$ & 55 & 348 & 58 & 665 & 685 \\
\hline \multirow[t]{2}{*}{1937} & Spring & Wheat & grain & 56 & 112 & 68 & 163 & 172 \\
\hline & & & straw & 101 & 241 & 120 & 354 & 393 \\
\hline 1938 & Potato & & & 931 & 1163 & 1235 & 1405 & 1414 \\
\hline \multirow[t]{2}{*}{1939} & Barley & grain & & 59 & 82 & 112 & 109 & 136 \\
\hline & & straw & & 231 & 325 & 292 & 401 & 486 \\
\hline \multirow[t]{2}{*}{1940} & Swede & roots & & 172 & 2488 & 379 & 4710 & 5275 \\
\hline & & tops & & 164 & 1640 & 180 & 2063 & 2408 \\
\hline \multirow[t]{2}{*}{1941} & Barley & grain & & 55 & 109 & 59 & 180 & 200 \\
\hline & & straw & & 364 & 455 & 396 & 416 & 421 \\
\hline 1942 & 1st yea & ar ley $h$ & hay & 348 & 630 & 460 & 636 & 608 \\
\hline 1943 & 2nd & $\Rightarrow$ & , & 340 & 713 & 368 & 778 & 895 \\
\hline 1944 & 3rd & , & • & 432 & 667 & 365 & 740 & 768 \\
\hline 1945 & 4th & , & • & 346 & 747 & 301 & 809 & 886 \\
\hline 1946 & 5 th & , & , & 113 & 530 & 112 & 590 & 639 \\
\hline 1947 & 6 th & , & - & 38 & 451 & 53 & 633 & 642 \\
\hline \multirow[t]{2}{*}{1948} & Oats & grain & & 114 & 332 & 147 & 373 & 385 \\
\hline & & straw & & 174 & 395 & 173 & 412 & 494 \\
\hline \multirow[t]{2}{*}{1949} & Oats & grain & & 238 & 352 & 340 & 417 & 401 \\
\hline & & straw & & 331 & 620 & 488 & 646 & 674 \\
\hline \multirow[t]{2}{*}{1950} & Barley & grain & & 91 & 232 & 116 & 274 & 311 \\
\hline & & straw & & 214 & 426 & 261 & 504 & 548 \\
\hline \multirow[t]{2}{*}{1951} & Oats & grain & & 104 & 300 & 118 & 316 & 315 \\
\hline & & straw & & 156 & 382 & 178 & 438 & 440 \\
\hline \multirow[t]{2}{*}{1952} & Oats & grain & & 123 & 366 & 182 & 382 & 345 \\
\hline & & straw & & 262 & 481 & 342 & 780 & 666 \\
\hline \multirow[t]{2}{*}{1953} & Barley & grain & & 40 & 96 & 72 & 70 & 92 \\
\hline & & straw & & 148 & 396 & 254 & 426 & 450 \\
\hline \multirow[t]{2}{*}{1954} & Oats & grain & & 112 & 206 & 123 & 202 & 236 \\
\hline & & straw & & 240 & 354 & 266 & 408 & 442 \\
\hline \multirow[t]{2}{*}{1955} & Barley & grain & & 84 & 288 & 121 & 365 & 362 \\
\hline & & straw & & 70 & 462 & 169 & 643 & 699 \\
\hline 1956 & No resu & ults & & & & & & \\
\hline \multirow[t]{2}{*}{1957} & Oats & grain & & 75 & 209 & 101 & 199 & 138 \\
\hline & & straw & & 130 & 352 & 151 & 386 & 361 \\
\hline \multirow[t]{2}{*}{1958} & Oats & grain & & 133 & 296 & 125 & 343 & 345 \\
\hline & & straw & & 299 & 642 & 270 & 942 & 888 \\
\hline
\end{tabular}


Appendix II. Annual crop yields in test. No. 2, $\mathrm{kg}$ per hectare $\mathrm{x} 10^{-1}$.

\begin{tabular}{|c|c|c|c|c|c|c|}
\hline \multirow[t]{2}{*}{ Year } & \multirow[t]{2}{*}{ Test plant } & \multicolumn{5}{|c|}{ Treatment } \\
\hline & & $\mathrm{P}$ & $\mathrm{PK}_{40}$ & $\mathrm{PK}_{20}$ & $\mathrm{P} 2 \mathrm{~K}_{40}$ & $\mathrm{P} 2 \mathrm{~K}_{20}$ \\
\hline 1933 & Potato & 759 & 932 & 937 & 1118 & 1068 \\
\hline 1934 & Potato & 2414 & 2676 & 2554 & 2591 & 2780 \\
\hline \multirow{2}{*}{1935} & Barley grain & 129 & 149 & 142 & 147 & 148 \\
\hline & straw & 441 & 492 & 479 & 491 & 487 \\
\hline 1936 & lst year ley hay & 575 & 666 & 753 & 731 & 763 \\
\hline \multicolumn{2}{|c|}{ Treatments changed } & $\mathrm{P}$ & $\begin{array}{r}\mathrm{PK} \\
\mathrm{K}_{2} \mathrm{SO}_{4}\end{array}$ & $\mathrm{PK}_{48}$ & $\begin{array}{r}\mathrm{P} 2 \mathrm{~K} \\
\mathrm{~K}_{2} \mathrm{SO}_{4}\end{array}$ & $\mathrm{P}_{2} \mathrm{~K}_{48}$ \\
\hline 1937 & 2nd year ley hay & 597 & 731 & 804 & 810 & 816 \\
\hline 1938 & 3rd & 285 & 575 & 578 & 632 & 622 \\
\hline 1939 & 4th, , & 216 & 435 & 463 & 525 & 567 \\
\hline 1940 & 5 th , , & 160 & 419 & 414 & 400 & 428 \\
\hline \multirow[t]{2}{*}{1941} & Oats grain & 290 & 225 & 277 & 213 & 231 \\
\hline & straw & 370 & 415 & 409 & 383 & 1384 \\
\hline 1942 & Potato & 968 & 1203 & 1290 & 1188 & 165 \\
\hline 1943 & No results & & & & & \\
\hline 1944 &,$\quad$ & & & & & \\
\hline \multirow[t]{2}{*}{1945} & Barley grain & 187 & 168 & 197 & 165 & 191 \\
\hline & straw & 532 & 525 & 560 & 517 & 558 \\
\hline \multirow[t]{2}{*}{1946} & Spring Wheat grain & 228 & 232 & 239 & 234 & 226 \\
\hline & straw & 433 & 462 & 466 & 471 & 470 \\
\hline \multirow[t]{2}{*}{1947} & Spring Wheat grain & 163 & 160 & 177 & 140 & 182 \\
\hline & straw & 442 & 478 & 497 & 517 & 489 \\
\hline 1948 & 1st year ley hay & 441 & 562 & 592 & 577 & 584 \\
\hline 1949 & 2nd, & 361 & 533 & 561 & 575 & 652 \\
\hline 1950 & 3rd , , & 396 & 742 & 786 & 817 & 856 \\
\hline 1951 & 4th, , & 482 & 828 & 805 & 827 & 885 \\
\hline \multirow[t]{2}{*}{1952} & Oats grain & 327 & 343 & 365 & 366 & 343 \\
\hline & straw & 586 & 680 & 785 & 744 & 732 \\
\hline 1953 & No results & & & & & \\
\hline \multirow[t]{2}{*}{1954} & Oats grain & 128 & 114 & 110 & 148 & 158 \\
\hline & straw & 284 & 340 & 392 & 352 & 366 \\
\hline 1955 & No results & & & & & \\
\hline 1956 & , $\quad$ & & & & & \\
\hline \multirow[t]{2}{*}{1957} & Oats grain & 284 & 258 & 241 & 223 & 223 \\
\hline & straw & 395 & 426 & 436 & 431 & 417 \\
\hline \multirow[t]{2}{*}{1958} & grain & 309 & 326 & 343 & 351 & 342 \\
\hline & straw & 519 & 613 & 608 & 554 & 645 \\
\hline
\end{tabular}




\section{REFERENCES}

(1) Antrinen, O. 1957. Saraturvesuon saveus- ja lannoituskokeen tuloksia. Referat: Ergebnisse eines Lehmzufuhr- und Düngungsversuchs auf Seggentorfmoor. Valt. maatal. koetoim. julk. 163: $1-20$.

(2) Hirvensalo, U. E. 1947. Nousevien kalimäärien vaikutuksista savetulla ja saveamattomalla mutasuolla. S. suovilj. yhd. vuosik. 50: 43-57.

(3) Malm, E. A. 1932. Nousevien kalimäärien vaikutuksesta sekä saveamattomalla että savetulla mutasuomaalla Leteensuon koeasemalla. Ibid. 35: 87-99.

(4) PESsı, Y. 1959. Kivennäismaan vaikutuksesta rahkasuon maanparannusaineena Leteensuon koeaseman pitkäaikaisten kenttäkokeiden perusteella. Summary: On the effect of mineral soil as a soil improving agent on Sphagnum bogs on the basis of prolonged field tests at Leteensuo Experimental Station. Acta agr. fenn. 94, 14: 241-268.

(5) $\rightarrow-1960$. Kivennäismaan merkityksestä mutasuon maanparannusaineena Leteensuon koeaseman pitkäaikaisten kenttäkokeiden perusteella. Summary: On the significance of mineral soils as a soil improving agent on fens on the basis of prolonged field tests at Leteensuo Experimental Station. Ibid. 95, 3: 1-27.

(6) Takala, M. 1959. Suoviljelysten fosfaattilannoituksesta. Summary: On phosphate fertilization of peat soils. S. Suovilj. yhd. vuosik. $63: 30-38$.

(7) Vesıkıvi, A. 1929. Suonsavetuksen ja -hiekoituksen taloudellisesta kannattavaisuudesta. Referat: Ưber die Rentabilität der Lehm- und Sandmischkultur auf Moorboden. S. suovilj. yhd. tiet. julk. 12: 1-131.

(8) - - Suonsaveuksen ja -hiekoituksen lannoitusvaikutuksesta. Referat: Úber die Düngewirkung der Lehm- und Sandmischungen auf Moorboden. Ibid. 14: 1-29.

S E L O S T S :

MUTASUON KALILANNOITUKSESTA LETEENSUON KOKEIDEN PERUSTEELLA

YRJö PESSI

Suoviljelysyhdistys, Leteensuon koeasema

Kalilannoitus on Leteensuon pitkäaikaisissa kokeissa lisännyt huomattavasti heinãsatoja sekä savetulla että saveamattomalla mutasuolla, jotka ovat saaneet fosfaattilannoituksen. Savella on ollut huomattava kalilannoitusvaikutus, joskin myös muita vaikutuksia, joita lannoituksella ei voitane korvata.

Kevätviljasatoihin kalilannoitus on vaikuttanut eri tavoin eri kokeissa. Kahdessa kokeessa se on lisännyt jyväsatoja, mutta ei yhdessä. Viimeksimainitun kokeen tulokseen lienee vaikuttanut tulvan koealueelle kuljettama liete.

Juurikasvien juurisatoa kalilannoitus on lisännyt huomattavasti.

Mikäli fosfaattilannoitusta ei ole annettu, on kalilannoituksen vaikutus jäănyt yleensä vähäiseksi tai lähes olemattomaksi kaikilla kasveilla.

Kalisuolan ja kaliumsulfaatin välinen eroavuus on ollut lähes olematon, joskin on todettava, ettei koekasvina ole ollut perunaa, jolla näiden lannoitteiden välinen ero tulisi ehkä parhaiten esiin. 